\title{
A Differential Approach for Bounding the Index of Graphs under Perturbations
}

\author{
C. Dalfó M.A. Fiol E. Garriga \\ Departament de Matemàtica Aplicada IV \\ Universitat Politècnica de Catalunya \\ $\{$ cdalfo,fiol, egarriga\}@ma4.upc.edu
}

Submitted: Mar 24, 2011; Accepted: Aug 22, 2011; Published: Sep 2, 2011

Mathematics Subject Classification: 05C50 (47A55).

\begin{abstract}
This paper presents bounds for the variation of the spectral radius $\lambda(G)$ of a graph $G$ after some perturbations or local vertex/edge modifications of $G$. The perturbations considered here are the connection of a new vertex with, say, $g$ vertices of $G$, the addition of a pendant edge (the previous case with $g=1$ ) and the addition of an edge. The method proposed here is based on continuous perturbations and the study of their differential inequalities associated. Within rather economical information (namely, the degrees of the vertices involved in the perturbation), the best possible inequalities are obtained. In addition, the cases when equalities are attained are characterized. The asymptotic behavior of the bounds obtained is also discussed. For instance, if $G$ is a connected graph and $G_{u}$ denotes the graph obtained from $G$ by adding a pendant edge at vertex $u$ with degree $\delta_{u}$, then,

$$
\lambda\left(G_{u}\right) \leq \lambda(G)+\frac{\delta_{u}}{\lambda^{3}(G)}+\mathrm{o}\left(\frac{1}{\lambda^{3}(G)}\right) .
$$
\end{abstract}

Keywords. Graph, Adjacency matrix, Spectral radius, Graph perturbation, Differential inequalities.

\section{Introduction}

When we represent a graph by its adjacency matrix, it is natural to ask how the properties of the graph are related to the spectrum of the matrix. As is well-known, the spectrum does not characterize the graph, that is, there are nonisomorphic cospectral graphs. However, important properties of the graph stem from the knowledge of its spectrum. A summary of these relations can be found in Schwenk and Wilson [12] and, in a 
more extensive way, in Cvetković, Doob and Sachs [3] and Cvetković, Doob, Gutman and Torgašev [2].

The perturbation of a graph $G$ is to be thought of as a local modification, such as the addition or deletion of a vertex or an edge. The cases studied here are the addition of a vertex (together with some incident edges), an edge and a pendant edge. When we make the perturbation, the spectrum changes and it is particulary interesting to study the behavior of the maximum eigenvalue $\lambda(G)$, which is called spectral radius or index of $G$. For a comprehensive survey of results about this parameter, we refer the reader to Cvetković and Rowlinson [4]. In particular, accurate bounds for $\lambda(G)$ were obtained, under some conditions, with the knowledge of the spectral radius, the associated eigenvector and the second eigenvalue. More details about these methods can be found in the survey by Rowlinson [10].

The bounds obtained here for the index of a perturbed graph are a bit less precise than those in Rowlinson [10], but we believe that ours have two aspects of interest. First, they are derived from a mere knowledge of the degrees of the vertices involved in the perturbation. Second, they are the best possible, in the sense that we characterize the cases in which the bounds are attained. Our approach is based on the study of some differential inequalities, seeing the perturbation as a continuous process or, to be more precise, as a linear matrix perturbation. Although the theory of matrix perturbations (see, for instance, the textbook by Stewart and Sun [13] or the chapter by Li [7]) has been commonly used in this context, to the authors' knowledge, our method has not been used before to bound the index of graphs under perturbations.

\section{Notation and Basic Concepts}

Our graphs are undirected, simple (without loops or multiple edges), connected and finite. The graph $G=(V, E)$ has set of vertices $V$, with cardinality $n=|V|$, and set of edges $E$. The trivial graph with only one vertex $u$ is denoted by $K_{1}=\{u\}$. If $G_{1}=\left(V_{1}, E_{1}\right)$ and $G_{2}=\left(V_{2}, E_{2}\right)$, then $G_{1} \cup G_{2}=\left(V_{1} \cup V_{2}, E_{1} \cup E_{2}\right)$ and $G_{1}+G_{2}=\left(V_{1} \cup V_{2}, E_{1} \cup E_{2} \cup E\right)$, where $E$ is the set of edges that join every vertex of $V_{1}$ with all vertices of $V_{2}$. The adjacency matrix $\boldsymbol{A}=\left(a_{i j}\right)$ of $G$ has entries $a_{i j}=1$ if $u_{i} u_{j} \in E$ and $a_{i j}=0$ otherwise. We denote by $\boldsymbol{j}$ the (column) vector of $\mathbb{R}^{n}$ with all its entries equal to 1 . Hence, $\boldsymbol{A} \boldsymbol{j}$ is the vector of degrees $\left(\delta_{1}, \delta_{2}, \ldots, \delta_{n}\right)^{\top}$. In particular, $G$ is regular of degree $\delta$ if and only if $\boldsymbol{A} \boldsymbol{j}=\delta \boldsymbol{j}$.

A real matrix $\boldsymbol{M}=\left(m_{i j}\right)$ is said to be nonnegative if $m_{i j} \geq 0$, for any $i, j$. We say that $\boldsymbol{M}$ is connected if, given any pair $i$ and $j$, there exists a sequence $i_{0}, i_{1}, \ldots, i_{r}$ such that $i_{0}=i, i_{r}=j$ and $m_{i_{h-1} i_{h}} \neq 0$, for $h=1,2, \ldots, r$. Trivially, the adjacency matrix of a connected graph is symmetric, nonnegative and connected.

The spectrum of a square matrix is the set of its eigenvalues in the complex plane. The spectral radius is the maximum of the modulus of its eigenvalues. If the matrix is the adjacency matrix of a graph, we call it the index of the graph. A symmetric real matrix has only real eigenvalues, which are numbered in nonincreasing order $\lambda_{1} \geq \lambda_{2} \geq \cdots \geq \lambda_{n}$. Then, the spectral radius is the maximum of $\left|\lambda_{1}\right|$ and $\left|\lambda_{n}\right|$. Also, the spectral radius can be defined as $\lambda=\sup \{\|\boldsymbol{A} \boldsymbol{x}\|:\|\boldsymbol{x}\|=1\}$, and defines a norm in the space of symmetric 
matrices. Then, $\|\boldsymbol{A} \boldsymbol{u}\| \leq\|\boldsymbol{A}\|\|\boldsymbol{u}\|$ for any vector $\boldsymbol{u}$, with equality if and only if $\boldsymbol{u}$ is an eigenvector associated with an eigenvalue giving the spectral radius. For a connected nonnegative symmetric real matrix, the theorem of Perron-Frobenius states the following:

1. The first eigenvalue equals the spectral radius $\lambda_{1}=\lambda$.

2. The eigenvalue $\lambda_{1}$ is a simple root of the characteristic polynomial.

3. There is a unitary eigenvector $\boldsymbol{x}$ corresponding to $\lambda_{1}$ with strictly positive entries.

\section{General Technique}

Let $\mathcal{S}^{+}$(respectively, $\mathcal{S}_{C}^{+}$) be the subset of symmetric, nonnegative (respectively, and connected) matrices of the space $M(n, n)$ of real $n \times n$ matrices.

When a perturbation modifies a graph into another, we denote by $G_{I}$ the initial graph and by $G_{F}$ the final graph. Similarly, if $\boldsymbol{A}_{I}$ and $\boldsymbol{A}_{F}$ are the adjacency matrices of the graphs $G_{I}$ and $G_{F}$ on $n$ vertices, we say that $\boldsymbol{A}_{F}$ is obtained from $\boldsymbol{A}_{I}$ by the perturbation $\boldsymbol{P}=\boldsymbol{A}_{F}-\boldsymbol{A}_{I}$.

If $G_{F}$ is connected, then the matrices $\boldsymbol{A}(t)=\boldsymbol{A}_{I}+t \boldsymbol{P}$ belong to $\mathcal{S}_{C}^{+}$for every $t \in(0,1]$. Similarly, if $G_{I}$ is connected, then $\boldsymbol{A}(t) \in \mathcal{S}_{C}^{+}$for $t \in[0,1)$. Also, if $G_{F}$ is connected and the perturbation matrix $\boldsymbol{P} \in \mathcal{S}^{+}$, then $\boldsymbol{A}_{I}+t \boldsymbol{P} \in \mathcal{S}_{C}^{+}$for $t \in(0, \infty)$.

If $\boldsymbol{A}$ and $\boldsymbol{P}$ are symmetric matrices, there exist continuous real functions $\mu_{1}(t), \mu_{2}(t)$, $\ldots, \mu_{n}(t)$, and continuous vectorial functions $\boldsymbol{x}_{1}(t), \boldsymbol{x}_{2}(t), \ldots, \boldsymbol{x}_{n}(t)$ that are, respectively, the eigenvalues of $\boldsymbol{A}(t)=\boldsymbol{A}+t \boldsymbol{P}$ and their associated eigenvectors. From the implicit

function theorem, if $\mu_{i}\left(t_{0}\right)$ is a simple eigenvalue, then $\mu_{i}$ is a $\mathcal{C}^{1}$-function in a neighborhood of $t_{0}$. Therefore, if $\boldsymbol{A}(t) \in \mathcal{S}_{C}^{+}$for $t$ belonging to an interval $I$, the spectral radius is a continuously differentiable function in $I$. In the three results that we present, the perturbation matrix $\boldsymbol{P}$ belongs to $\mathcal{S}^{+}$and the perturbed matrix $\boldsymbol{A}_{F}=\boldsymbol{A}_{I}+\boldsymbol{P}$ to $\mathcal{S}_{C}^{+}$. Thus, the normalized positive eigenvector $\boldsymbol{x}(t)$ associated with the spectral radius $\lambda(t)$ of the matrix $\boldsymbol{A}(t)=\boldsymbol{A}_{I}+t \boldsymbol{P}$ is a $\mathcal{C}^{1}(0, \infty)$-function, which can be extended with continuity to $[0, \infty)$, but now $\boldsymbol{x}(t)$ might have lost the strictly positive character of its entries.

Our technique is based on the following result:

Lemma 3.1 Let $\boldsymbol{x}(t)=\left(\alpha_{1}, \alpha_{2}, \ldots, \alpha_{n}\right)^{\top}$ be the normalized $\lambda(t)$-eigenvector of the matrix $\boldsymbol{A}(t)=\boldsymbol{A}_{I}+t \boldsymbol{P}$ with $\boldsymbol{P}=\left(p_{i j}\right)$. Then,

$$
\lambda^{\prime}=\langle\boldsymbol{P} \boldsymbol{x}, \boldsymbol{x}\rangle=\sum_{i, j=1}^{n} p_{i j} \alpha_{i} \alpha_{j} .
$$

Proof. By differentiating the expression $\boldsymbol{A x}=\left(\boldsymbol{A}_{I}+t \boldsymbol{P}\right) \boldsymbol{x}=\lambda \boldsymbol{x}$, we get

$$
\boldsymbol{P} \boldsymbol{x}+\boldsymbol{A} \boldsymbol{x}^{\prime}=\lambda^{\prime} \boldsymbol{x}+\lambda \boldsymbol{x}^{\prime} .
$$

Then, the result follows by taking the inner product by $\boldsymbol{x}$ and observing that, from $\langle\boldsymbol{x}, \boldsymbol{x}\rangle=1$, we have $\left\langle\boldsymbol{x}^{\prime}, \boldsymbol{x}\right\rangle=0$ and $\left\langle\boldsymbol{A} \boldsymbol{x}^{\prime}, \boldsymbol{x}\right\rangle=\left\langle\boldsymbol{x}^{\prime}, \boldsymbol{A} \boldsymbol{x}\right\rangle=\lambda\left\langle\boldsymbol{x}^{\prime}, \boldsymbol{x}\right\rangle=0$. 
A first remark is that if $\boldsymbol{P} \in \mathcal{S}^{+}$and $\boldsymbol{A}_{F}=\boldsymbol{A}_{I}+\boldsymbol{P} \in \mathcal{S}_{C}^{+}$, then the spectral radius increases strictly and, in particular, $\lambda_{I}=\lambda(0)<\lambda(1)=\lambda_{F}$. Also, since there exists $\lim _{t \rightarrow 0^{+}} \lambda(t)=\sum_{i, j=1}^{n} p_{i j} \alpha_{i}(0) \alpha_{j}(0)$, by the mean value theorem, we have that $\lambda$ is also differentiable at 0 with $\lambda^{\prime}(0)=\sum_{i, j=1}^{n} p_{i j} \alpha_{i}(0) \alpha_{j}(0)$.

We present three results of bounds of the index of a graph for the following perturbations: connecting an isolated vertex, adding an edge and adding a pendant edge. Starting from Eq. (1), we give differential inequalities with information on the degrees of the vertices involved, and we characterize the case when they become equations. Solving these equations, we reach our conclusions by using the following result on differential inequalities (see Szarski [14]):

Lemma 3.2 Let $A$ be an open convex subset of $\mathbb{R}^{2}$ and let $f: A \rightarrow \mathbb{R},(t, x) \mapsto f(t, x)$,

be a continuous function with $\frac{\partial f}{\partial x}$ continuous. Let $u, v:\left[t_{0}, \alpha\right) \rightarrow \mathbb{R}$ be continuously differentiable functions, such that:

1. For all $t \in\left[t_{0}, \alpha\right),(t, u(t)) \in A,(t, v(t)) \in A$.

2. Function $u$ satisfies: $u^{\prime}(t)=f(t, u(t))$ for all $t \in\left[t_{0}, \alpha\right), u\left(t_{0}\right)=x_{0}$.

3. Function $v$ satisfies: $v^{\prime}(t)<f(t, v(t))$ for all $t \in\left(t_{0}, \alpha\right), v\left(t_{0}\right)=x_{0}, v^{\prime}\left(t_{0}\right) \leq$ $f\left(t_{0}, v\left(t_{0}\right)\right)$.

Then, $v(t)<u(t)$ for all $\in\left(t_{0}, \alpha\right)$.

\section{Connection of an isolated vertex}

Our first result is on the change of the index of a graph when we connect an isolated vertex to some other vertices. For this case Rowlinson [11] computed the characteristic polynomial of the modified graph in terms of the characteristic polynomial of the initial graph and some entries of its idempotents (see also Cvetković and Rowlinson [5, p.90] for a shorter proof).

Theorem 4.1 Let $G_{I}=(V, E)$ be a graph with $|V| \geq 2$ and an isolated vertex $u$. Given some vertices $v_{1}, v_{2}, \ldots, v_{g}$ different from $u$, we denote by $G_{F}$ the graph $(V, E \cup$ $\left.\left\{u v_{1}, u v_{2}, \ldots, u v_{g}\right\}\right)$, which is assumed to be connected. If $\lambda_{I}$ and $\lambda_{F}$ are the spectral radii of $G_{I}$ and $G_{F}$, respectively, then the following inequality holds:

$$
\lambda_{F} \leq H^{-1}\left(\lambda_{I}\right)
$$

where the function $H:(0,+\infty) \rightarrow \mathbb{R}$ is defined by $H(\xi)=\xi-\frac{g}{\xi}$. The equality is satisfied if and only if $G_{F}=\{u\}+G$, with $G$ being a regular graph.

Proof. Let $n+1$ be the order of the graphs $G_{I}$ and $G_{F}$. The continuous perturbation of the matrix associated with $G_{I}$ that produces the matrix associated with $G_{F}$ can be 
described by

$$
\boldsymbol{A}(t)=\boldsymbol{A}_{I}+t \boldsymbol{P}=\left(\begin{array}{cccc}
0 & 0 & \cdots & 0 \\
0 & & & \\
\vdots & & \boldsymbol{C} & \\
0 & &
\end{array}\right)+t\left(\begin{array}{cccc}
0 & \cdots & \boldsymbol{w}^{\top} & \cdots \\
\vdots & & & \\
\boldsymbol{w} & & \boldsymbol{O} & \\
\vdots & & &
\end{array}\right), \quad t \in[0,1]
$$

where $\boldsymbol{w}$ is the column binary vector associated with the perturbation and $\boldsymbol{C}$ is the adjacency matrix of the graph $G=G_{I}-\{u\}$. Note that, for any $t \in(0,1]$, the matrix $\boldsymbol{A}(t)$ is nonnegative and connected. Let $\lambda(t)$ be the spectral radius of $\boldsymbol{A}(t)$. Let $\boldsymbol{x}(t)=$ $(\alpha \mid \boldsymbol{z})^{\top}=\left(\alpha, z_{1}, z_{2}, \ldots, z_{n}\right)^{\top}$ be its normalized positive eigenvector. Then, by Eq. (1),

$$
\lambda^{\prime}=\langle\boldsymbol{P} \boldsymbol{x}, \boldsymbol{x}\rangle=2 \alpha\langle\boldsymbol{z}, \boldsymbol{w}\rangle .
$$

From $\boldsymbol{A}(t) \boldsymbol{x}(t)=\lambda(t) \boldsymbol{x}(t)$, we have

$$
\left(\begin{array}{cc}
0 & t \boldsymbol{w}^{\top} \\
t \boldsymbol{w} & \boldsymbol{C}
\end{array}\right)\left(\begin{array}{c}
\alpha \\
\boldsymbol{z}
\end{array}\right)=\left(\begin{array}{c}
t\langle\boldsymbol{w}, \boldsymbol{z}\rangle \\
t \alpha \boldsymbol{w}+\boldsymbol{C} \boldsymbol{z}
\end{array}\right)=\left(\begin{array}{c}
\lambda \alpha \\
\lambda \boldsymbol{z}
\end{array}\right)
$$

and the first scalar equation gives

$$
\lambda^{2} \alpha^{2}=t^{2}\langle\boldsymbol{z}, \boldsymbol{w}\rangle^{2} \leq t^{2}\|\boldsymbol{z}\|^{2} g=t^{2}\left(1-\alpha^{2}\right) g .
$$

Hence,

$$
\lambda^{\prime}=2 \lambda \frac{\alpha^{2}}{t} \leq \frac{2 g t \lambda}{\lambda^{2}+g t^{2}} .
$$

The inequalities (3) and (4) are either equalities or strict inequalities in the whole interval $(0,1]$. Indeed, if the equalities are satisfied for $t_{0}$, then $\boldsymbol{z}\left(t_{0}\right)$, which has only positive entries, would be proportional to $\boldsymbol{w}$, which is not null. Therefore, $\boldsymbol{w}=\boldsymbol{j}$ and $\boldsymbol{z}\left(t_{0}\right)=\beta \boldsymbol{j}$. Hence, at $t=t_{0}$ the last $n$ equations of (2) become $\boldsymbol{C} \boldsymbol{j}=\left(\lambda-t_{0} \frac{\alpha}{\beta}\right) \boldsymbol{j}$, where $\alpha=\alpha\left(t_{0}\right)$, so that $G_{I}=\{u\} \cup G, G_{F}=\{u\}+G$, and $G$ is a regular graph. To conclude that, in this situation, (4) is an equality for all $t \in(0,1]$, let us study the existence of solutions to the following system:

$$
\left(\begin{array}{cccc}
0 & t & \cdots & t \\
t & & \\
\vdots & \boldsymbol{C} & \\
t & &
\end{array}\right)\left(\begin{array}{c}
\alpha \\
\beta \\
\vdots \\
\beta
\end{array}\right)=\lambda\left(\begin{array}{c}
\alpha \\
\beta \\
\vdots \\
\beta
\end{array}\right), \quad \alpha^{2}+n \beta^{2}=1
$$

Then, for all $t$, we obtain the solution:

$$
\lambda=\frac{\delta}{2}+\sqrt{\frac{\delta^{2}}{4}+n t^{2}}, \quad \alpha=\sqrt{\frac{\lambda-\delta}{2 \lambda-\delta}}, \quad \beta=\sqrt{\frac{\lambda}{n(2 \lambda-\delta)}},
$$


where $\delta=\lambda-t \frac{\alpha}{\beta}$ denotes the degree of $G$. Notice that, in fact, $\lambda$ is the largest eigenvalue of the quotient matrix

$$
\left(\begin{array}{cc}
0 & n t \\
t & \delta
\end{array}\right)
$$

corresponding to an equitable (or regular) partition (see Godsil [6]).

Now we have the following cases, where $f(t, \lambda)=\frac{2 g t \lambda}{\lambda^{2}+g t^{2}}$ :

(a) $\lambda^{\prime}=f(t, \lambda)$ for all $t \in[0,1], \lambda(0)=\lambda_{I}$, if $G_{F}=\{u\}+G$, with $G$ being a regular graph.

(b) $\lambda^{\prime}<f(t, \lambda)$ for all $t \in(0,1], \lambda^{\prime}(0)=f(0, \lambda(0)), \lambda(0)=\lambda_{I}$, in any other case.

The Cauchy problem

$$
y^{\prime}=\frac{2 g t y}{y^{2}+g t^{2}}, \quad y(0)=\lambda_{I},
$$

can be solved by making the changes $y=\sqrt{R S}$ and $t=\sqrt{S}$, so giving

$$
y^{2}(t)-\lambda_{I} y(t)-g t^{2}=0 \text {. }
$$

Hence,

$$
y(1)-\frac{g}{y(1)}=\lambda_{F}-\frac{g}{\lambda_{F}}=\lambda_{I}
$$

and, introducing the bijection $H:(0,+\infty) \rightarrow \mathbb{R}, H(\xi)=\xi-\frac{g}{\xi}$, the theorem follows from Lemma 3.2.

In fact, as commented by one of the referees, this result can be also obtained from the mentioned result of Rowlinson [11] which, using our notation, reads as follows: Let $\mu_{0}>\mu_{1}>\cdots>\mu_{d}$ be the distinct eigenvalues of $G$ (so that $\mu_{0}=\lambda_{I}$ ) and, for every $i=0,1, \ldots, d$, let $\boldsymbol{E}_{i}$ be the (principal) idempotent corresponding to the orthogonal projection of $\mathbb{R}^{n}$ onto the eigenspace $\mathcal{E}\left(\mu_{i}\right)$. Then, the characteristic polynomials $\phi_{G_{F}}$ and $\phi_{G}$ of the corresponding graphs satisfy

$$
\phi_{G_{F}}(x)=\phi_{G}(x)\left(x-\sum_{i=0}^{d} \frac{\left\|\boldsymbol{E}_{i} \boldsymbol{w}\right\|^{2}}{x-\mu_{i}}\right) .
$$

In our case, for $x=\lambda_{F}$, the formula gives

$$
\lambda_{F}=\sum_{i=0}^{d} \frac{\left\|\boldsymbol{E}_{i} \boldsymbol{w}\right\|^{2}}{\lambda_{F}-\mu_{i}} \leq \frac{\left\|\boldsymbol{E}_{0} \boldsymbol{w}\right\|^{2}}{\lambda_{F}-\mu_{0}} \leq \frac{g}{\lambda_{F}-\lambda_{I}},
$$

whence $H\left(\lambda_{F}\right) \leq \lambda_{I}$. Moreover, if equality holds then $\boldsymbol{E}_{i} \boldsymbol{w}=\mathbf{0}$ for $i=1, \ldots, d$, whence $\boldsymbol{w} \in \mathcal{E}\left(\mu_{0}\right)$. Consequently, if $G$ has, say, $k$ components, $\boldsymbol{w}$ must be a linear combination of their associated characteristic vectors, $\boldsymbol{c}_{1}, \boldsymbol{c}_{2}, \ldots, \boldsymbol{c}_{k}$, and, since $G_{F}$ is connected, necessarily $\boldsymbol{w}=\sum_{j=1}^{k} \boldsymbol{c}_{j}=\boldsymbol{j}$. 


\section{Addition of an edge}

The second result that we present is on the change of the index when we add an edge to a graph. In this context, Rowlinson [9] proved that, under some conditions, the index of the perturbed graph can be determined by the eigenvalues of the original graph together with some of its angles. Moreover, some upper and lower bounds for such an index were given by Maas [8].

Theorem 5.1 Let $G_{I}=(V, E)$ be a graph with $|V| \geq 3$ and $E \neq \emptyset$, and let $u, v \in V$ be two nonadjacent vertices with degrees $\delta_{u}, \delta_{v}$. Let $G_{F}=(V, E \cup\{u v\})$, which we assume to be connected. If $\lambda_{I}$ and $\lambda_{F}$ are, respectively, the indices of $G_{I}$ and $G_{F}$, then

$$
\lambda_{F} \leq 1+K^{-1}\left(K\left(\lambda_{I}\right)-1\right)
$$

where $K:(0, \infty) \rightarrow \mathbb{R}$ is defined as $K(\xi)=\xi-\frac{\delta_{u}+\delta_{v}}{\xi}$. The equality is satisfied if and only if $G_{I}=(\{u\} \cup\{v\})+G$, where $G$ is a regular graph.

Proof. Let $n+2$ be the order of graphs $G_{I}$ and $G_{F}$ with adjacency matrices $\boldsymbol{A}_{I}$ and $\boldsymbol{A}_{F}$, respectively. In the language of perturbations, we can consider that $\boldsymbol{A}_{I}$ and $\boldsymbol{A}_{F}$ are related by $\boldsymbol{A}_{F}=\boldsymbol{A}_{I}+\boldsymbol{P}$, where $\boldsymbol{P}=\left(p_{i j}\right)$ has entries $p_{12}=p_{21}=1$ and $p_{i j}=0$ otherwise (if necessary, we rearrange the vertices so that $v_{1}=u$ and $v_{2}=v$ ). Considering the continuous perturbation, let us consider the uniparametric family of matrices

$$
\boldsymbol{A}(t)=\boldsymbol{A}_{I}+t \boldsymbol{P}=\left(\begin{array}{ccccc}
0 & t & \cdots & \boldsymbol{w}_{u}^{\top} & \cdots \\
t & 0 & \cdots & \boldsymbol{w}_{v}^{\top} & \cdots \\
\vdots & \vdots & & & \\
\boldsymbol{w}_{u} & \boldsymbol{w}_{v} & & \boldsymbol{C} & \\
\vdots & \vdots & & &
\end{array}\right), \quad t \in[0,1]
$$

where $\boldsymbol{w}_{u}, \boldsymbol{w}_{v} \in\{0,1\}^{n}$ and $\boldsymbol{C}$ is the $n \times n$ adjacency matrix of the subgraph $G_{I}-\{u\}-\{v\}$.

Let $\lambda(t)$ be the spectral radius of $\boldsymbol{A}(t)$, which is a continuous function on $t$ for $t \in[0,1]$, and is differentiable for $t \in(0,1]$ by the connectedness of $\boldsymbol{A}(t)$.

Now, with $\boldsymbol{x}(t)=(\alpha, \beta \mid \boldsymbol{z})^{\top}=\left(\alpha, \beta, z_{1}, z_{2}, \ldots, z_{n}\right)^{\top}$, Eq. (1) becomes

$$
\lambda^{\prime}=\langle\boldsymbol{P} \boldsymbol{x}, \boldsymbol{x}\rangle=2 \alpha \beta .
$$

Considering the first two entries of $(\lambda(t) \boldsymbol{I}-\boldsymbol{A}(t)) \boldsymbol{x}(t)=\mathbf{0}$, we get the system

$$
\boldsymbol{M}\left(\begin{array}{c}
\alpha \\
\beta
\end{array}\right)=\left(\begin{array}{c}
r \\
s
\end{array}\right)
$$

with

$$
\boldsymbol{M}=\left(\begin{array}{cc}
\lambda & -t \\
-t & \lambda
\end{array}\right), \quad r=\left\langle\boldsymbol{w}_{u}, \boldsymbol{z}\right\rangle, \quad s=\left\langle\boldsymbol{w}_{v}, \boldsymbol{z}\right\rangle
$$


Introducing the angles $\varphi_{u}$ and $\varphi_{v}$ that the vectors $\boldsymbol{w}_{u}$ and $\boldsymbol{w}_{v}$ form with $\boldsymbol{z}$, we can write

$$
\begin{aligned}
\alpha^{2}+\beta^{2} & =\left\|\boldsymbol{M}^{-1}\left(\begin{array}{c}
r \\
s
\end{array}\right)\right\|^{2} \leq\left\|\boldsymbol{M}^{-1}\right\|^{2}\left(r^{2}+s^{2}\right) \\
& =\|\boldsymbol{z}\|^{2} \frac{\left(\delta_{u} \cos ^{2} \varphi_{u}+\delta_{v} \cos ^{2} \varphi_{v}\right)}{(\lambda-t)^{2}} \\
& \leq \frac{1-\alpha^{2}-\beta^{2}}{(\lambda-t)^{2}}\left(\delta_{u}+\delta_{v}\right),
\end{aligned}
$$

since $\frac{1}{\lambda-t}$ is the maximum eigenvalue of $\boldsymbol{M}^{-1}$ with associated eigenvector $(1,1)$. (Note that $\boldsymbol{M}$ is always invertible since, from the hypotheses, $\lambda(t)>\lambda(0) \geq 1$ for $t \in(0,1]$.) Then,

$$
2 \alpha \beta \leq \alpha^{2}+\beta^{2} \leq \frac{\delta_{u}+\delta_{v}}{(\lambda-t)^{2}+\delta_{u}+\delta_{v}} .
$$

Therefore, the spectral radius of $\boldsymbol{A}(t)$ satisfy the following differential inequality:

$$
\lambda^{\prime} \leq \frac{\delta_{u}+\delta_{v}}{(\lambda-t)^{2}+\delta_{u}+\delta_{v}}, \quad \lambda(0)=\lambda_{I} .
$$

We now prove that, in the interval $(0,1]$, expression $(7)$ is always an equality or a strict inequality. Let us assume that there exists $t_{0} \in(0,1]$ such that $(7)$ is an equality. Observing (6), we see that the first inequality is equivalent to $\alpha=\beta$ and the second one to both equalities in (5). The first one occurs if $\sqrt{\delta_{u}} \cos \varphi_{u}=\sqrt{\delta_{v}} \cos \varphi_{v}$ and the second if $\cos \varphi_{u}=\cos \varphi_{v}=1$. Therefore, the equality in (7) is valid for a value $t_{0}$ when the following conditions are simultaneously satisfied:

$$
\delta_{u}=\delta_{v}, \quad \cos \varphi_{u}=\cos \varphi_{v}=1, \quad \alpha=\beta .
$$

As all the entries of $\boldsymbol{z}$ are different from zero and $\boldsymbol{w}_{u}, \boldsymbol{w}_{v}$ are not null vectors, then it follows that $\boldsymbol{w}_{u}=\boldsymbol{w}_{v}=\boldsymbol{j}$ and $\boldsymbol{x}\left(t_{0}\right)=(\alpha, \alpha, \gamma, \stackrel{(n)}{.}, \gamma)^{\top}$. The last $n$ entries of $\boldsymbol{A}\left(t_{0}\right) \boldsymbol{x}\left(t_{0}\right)=\lambda \boldsymbol{x}\left(t_{0}\right)$ give $2 \alpha \boldsymbol{j}+\gamma \boldsymbol{C} \boldsymbol{j}=\lambda \gamma \boldsymbol{j}$, that is,

$$
\boldsymbol{C} \boldsymbol{j}=\left(\lambda-2 \frac{\alpha}{\gamma}\right) \boldsymbol{j}
$$

which means that $G_{I}=(\{u\} \cup\{v\})+G$, with $G$ being a regular graph with adjacency matrix $\boldsymbol{C}$. Therefore, there exist positive integers $\alpha, \gamma$, such that, for all $t \in(0,1]$, $(\alpha, \alpha, \gamma, \stackrel{(n)}{.}, \gamma)^{\top}$ is an eigenvector (since all its entries are positive, it corresponds to the spectral radius). Indeed, the system

$$
\left(\begin{array}{ccccc}
0 & t & \cdots & \boldsymbol{j}^{\top} & \cdots \\
t & 0 & \cdots & \boldsymbol{j}^{\top} & \cdots \\
\vdots & \vdots & & & \\
\boldsymbol{j} & \boldsymbol{j} & & \boldsymbol{C} & \\
\vdots & \vdots & & &
\end{array}\right)\left(\begin{array}{c}
\alpha \\
\alpha \\
\gamma \\
\vdots \\
\gamma
\end{array}\right)=\lambda\left(\begin{array}{c}
\alpha \\
\alpha \\
\gamma \\
\vdots \\
\gamma
\end{array}\right), \quad 2 \alpha^{2}+n \gamma^{2}=1
$$


has solution

$$
\begin{aligned}
\alpha & =\frac{1}{2} \sqrt{1-\frac{\delta-t}{\sqrt{(\delta-t)^{2}+8 n}}} \\
\gamma & =\frac{1}{\sqrt{2 n}} \sqrt{1+\frac{\delta-t}{\sqrt{(\delta-t)^{2}+8 n}}} \\
\lambda & =\frac{1}{2}\left(t+\delta+\sqrt{(\delta-t)^{2}+8 n}\right)
\end{aligned}
$$

where $\delta$ is the degree of $G$, and inequalities (5) and (6) are equalities for all $t \in(0,1]$. Note that, as before, $\lambda$ corresponds to the largest eigenvalue of a quotient matrix, namely,

$$
\left(\begin{array}{ll}
t & n \\
2 & \delta
\end{array}\right)
$$

Extending by continuity to $[0,1]$, we have the following possibilities:

(a) $\lambda^{\prime}=f(t, \lambda)$, for all $t \in[0,1], \lambda(0)=\lambda_{I}$ if $G_{I}=(\{u\} \cup\{v\})+G$, with $G$ being regular;

(b) $\lambda^{\prime}<f(t, \lambda)$, for all $t \in(0,1], \lambda^{\prime}(0) \leq f(0, \lambda(0)), \lambda(0)=\lambda_{I}$, in any other case;

where $f$ is the right side of differential inequality (7).

Now, the solution to Cauchy's problem

$$
y^{\prime}=\frac{\delta_{u}+\delta_{v}}{(y-t)^{2}+\delta_{u}+\delta_{v}}, \quad y(0)=\lambda_{I}
$$

is

$$
y-\frac{\delta_{u}+\delta_{v}}{y-t}=\lambda_{I}-\frac{\delta_{u}+\delta_{v}}{\lambda_{I}} .
$$

By introducing the invertible function

$$
K:(0, \infty) \rightarrow \mathbb{R}, \quad K(\xi)=\xi-\frac{\delta_{u}+\delta_{v}}{\xi},
$$

we can write $y(1)=1+K^{-1}\left(K\left(\lambda_{I}\right)-1\right)$.

Lemma 3.2 applied to case $(b)$ completes the proof.

\section{Addition of a pendant edge}

The last result presented here is on the change of the index of a graph $G$ when we add a pendant edge to one of its vertices. In this context, Bell and Rowlinson [1] derived, under certain conditions, exact values for the index of the perturbed graph in terms of the spectrum and certain angles of $G$. 
Theorem 6.1 Let $G_{I}=(V, E)$ be a connected graph, let $u \in V$ be a vertex of degree $\delta_{u}$ and take a vertex $v \notin V$. Let $G_{F}=(V \cup\{v\}, E \cup\{u v\})$. If $\lambda_{I}$ and $\lambda_{F}$ are the spectral radii of $G_{I}$ and $G_{F}$ respectively, then

$$
\lambda_{F} \leq L_{2}^{-1} L_{1}\left(\lambda_{I}\right)
$$

where $L_{1}:(0,+\infty) \rightarrow \mathbb{R}$ is $L_{1}(\xi)=\xi-\frac{\delta_{u}}{\xi}$ and $L_{2}:(1,+\infty) \rightarrow \mathbb{R}$ is $L_{2}(\xi)=\xi-\frac{\delta_{u}}{\xi-\frac{1}{\xi}}$. The equality is satisfied if and only if $G_{I}=\{u\}+G$, with $G$ being a regular graph.

Proof. Let $n+1$ be the order of $G_{I}$. Rearranging the vertices suitably, the perturbation matrix $\boldsymbol{P}=\left(p_{i j}\right)$ has $p_{12}=p_{21}=1$ and the other entries are zero. Let us consider the matrices

$$
\boldsymbol{A}(t)=\left(\begin{array}{ccccc}
0 & t & 0 & \cdots & 0 \\
t & 0 & \cdots & \boldsymbol{w}^{\top} & \cdots \\
0 & \vdots & & & \\
\vdots & \boldsymbol{w} & & \boldsymbol{C} & \\
0 & \vdots & & &
\end{array}\right), \quad t \in[0,1],
$$

such that $\boldsymbol{A}(0)$ is the adjacency matrix of the graph $G_{I} \cup\{v\}$, with the same spectral radius as $G_{I}$.

Now Eq. (1) becomes

$$
\lambda^{\prime}=\langle\boldsymbol{P} \boldsymbol{x}, \boldsymbol{x}\rangle=2 \alpha \beta,
$$

where $\boldsymbol{x}(t)=(\alpha, \beta \mid \boldsymbol{z})^{\top}$, with $\boldsymbol{z}^{\top}=\left(z_{1}, z_{2}, \ldots, z_{n}\right)^{\top}$ being the normalized positive eigenvector, $t \in(0,1)$. The first two entries of the matrix equation $(\lambda(t) \boldsymbol{I}-\boldsymbol{A}(t)) \boldsymbol{x}(t)=\mathbf{0}$ give the system

$$
\begin{aligned}
\lambda \alpha-t \beta & =0, \\
-t \alpha+\lambda \beta & =\langle\boldsymbol{w}, \boldsymbol{z}\rangle .
\end{aligned}
$$

Introducing the angle $\varphi$ determined by $\boldsymbol{z}$ and $\boldsymbol{w}$, we can express the solution by

$$
\begin{aligned}
\alpha & =\sqrt{\delta_{u}} \frac{\|\boldsymbol{z}\|}{\lambda^{2}-t^{2}} t \cos \varphi, \\
\beta & =\sqrt{\delta_{u}} \frac{\|\boldsymbol{z}\|}{\lambda^{2}-t^{2}} \lambda \cos \varphi .
\end{aligned}
$$

Hence, using $\alpha^{2}+\beta^{2}+\|\boldsymbol{z}\|^{2}=1$, we obtain

$$
\lambda^{\prime}=\frac{2 \delta_{u} t \lambda \cos ^{2} \varphi}{\delta_{u}\left(\lambda^{2}+t^{2}\right) \cos ^{2} \varphi+\left(\lambda^{2}-t^{2}\right)^{2}} .
$$

The constraint $\cos ^{2} \varphi \leq 1$ implies that

$$
\lambda^{\prime} \leq \frac{2 \lambda t \delta_{u}}{\left(\lambda^{2}-t^{2}\right)^{2}+\delta_{u}\left(\lambda^{2}+t^{2}\right)}
$$


for all $t \in(0,1]$. Let us observe that the continuous extension of (8) to $t=0$ gives an equality, since $\alpha(0)=0$.

We now prove that inequality (8) is either an equality or a strict inequality in the interval $(0,1]$. Indeed, if there existed $t_{0} \in(0,1]$ for which $(8)$ were an equality, then $\boldsymbol{z}\left(t_{0}\right)$ and $\boldsymbol{w}$ would be proportional. As all the entries of $\boldsymbol{z}$ are strictly positive and $\boldsymbol{w}$ is not a null vector, then $\boldsymbol{w}=\boldsymbol{j}$ and $\boldsymbol{z}\left(t_{0}\right)=\delta \boldsymbol{j}$. The last $n$ equations of $\left(\lambda\left(t_{0}\right) \boldsymbol{I}-\boldsymbol{A}\left(t_{0}\right)\right) \boldsymbol{x}\left(t_{0}\right)=\mathbf{0}$ give $\boldsymbol{C} \boldsymbol{j}=\left(\lambda-\frac{\beta}{\delta}\right) \boldsymbol{j}$. Therefore, the graph $G_{I}$ is $\{u\}+G$, with $G$ being a regular graph of degree $\delta=\lambda-\frac{\beta}{\delta}$ and with adjacency matrix $\boldsymbol{C}$. Then, $\boldsymbol{z}=\delta \boldsymbol{j}$ and, therefore, it is proportional to $\boldsymbol{w}=\boldsymbol{j}$, for all $t \in(0,1]$. Indeed, the system

$$
\left(\begin{array}{ccccc}
0 & t & 0 & \cdots & 0 \\
t & 0 & \cdots & j^{\top} & \cdots \\
0 & \vdots & & & \\
\vdots & j & & C & \\
0 & \vdots & & &
\end{array}\right)\left(\begin{array}{c}
\alpha \\
\beta \\
\gamma \\
\vdots \\
\gamma
\end{array}\right)=\lambda\left(\begin{array}{c}
\alpha \\
\beta \\
\gamma \\
\vdots \\
\gamma
\end{array}\right), \quad \alpha^{2}+\beta^{2}+n \gamma^{2}=1
$$

gives the eigenvector of strictly positive entries

$$
\alpha=\frac{(\lambda-\delta) t}{\sqrt{\Lambda}}, \quad \beta=\frac{(\lambda-\delta) \lambda}{\sqrt{\Lambda}}, \quad \gamma=\frac{\lambda}{\sqrt{\Lambda}},
$$

where $\Lambda=2\left(n+t^{2}\right) \lambda^{2}-\delta\left(n+t+3 t^{2}\right) \lambda+2 t^{2} \delta^{2}$ and $\lambda$ is the maximum root of the polynomial $\lambda^{3}-\delta \lambda^{2}-\left(n+t^{2}\right) \lambda+\delta t^{2}$, which is the characteristic polynomial of the quotient matrix

$$
\left(\begin{array}{lll}
0 & t & 0 \\
t & 0 & n \\
0 & 1 & \delta
\end{array}\right)
$$

corresponding to an equitable partition.

By continuity, we thus have the two following possibilities:

(a) $\lambda^{\prime}=f(t, \lambda)$, for all $t \in[0,1], \lambda(0)=\lambda_{I}$ if $G_{I}=\{u\}+G$, with $G$ being a regular graph,

(b) $\lambda^{\prime}<f(t, \lambda)$, for all $t \in(0,1], \lambda^{\prime}(0) \leq f(0, \lambda(0)), \lambda(0)=\lambda_{I}$, in any other case,

where $f$ is the right side of differential inequality (8).

The differential equation

$$
y^{\prime}=2 \delta_{u} \frac{t y}{\left(y^{2}-t^{2}\right)^{2}+\delta_{u}\left(y^{2}+t^{2}\right)},
$$

with initial condition $y(0)=\lambda_{I}$, is transformed into a linear equation by means of the changes $y=\sqrt{\frac{R+S}{2}}, t=\sqrt{\frac{R-S}{2}}$. Solving it, we calculate implicitly $y(1)$, represented by $\nu$, as one root of the equation

$$
\left(\nu^{2}+1\right)\left(\nu^{2}-1-\delta_{u}\right)^{2}+\left(\nu^{2}-1\right)^{3}+2\left(\delta_{u}-\lambda_{I}^{2}-\frac{\delta_{u}^{2}}{\lambda_{I}^{2}}\right)\left(\nu^{2}-1\right)^{2}+\delta_{u}^{2}\left(\nu^{2}-1\right)=0,
$$


which may be factorized into the following two cubic equations:

$$
\begin{aligned}
& \nu^{3}-\left(\lambda_{I}-\frac{\delta_{u}}{\lambda_{I}}\right) \nu^{2}-\left(\delta_{u}+1\right) \nu+\left(\lambda_{I}-\frac{\delta_{u}}{\lambda_{I}}\right)=0, \\
& \nu^{3}+\left(\lambda_{I}-\frac{\delta_{u}}{\lambda_{I}}\right) \nu^{2}-\left(\delta_{u}+1\right) \nu-\left(\lambda_{I}-\frac{\delta_{u}}{\lambda_{I}}\right)=0 .
\end{aligned}
$$

The three roots of both equations are real, but only one in the first equation satisfies the necessary condition $\nu \geq \sqrt{\delta_{u}+1}$. Introducing the bijective functions

$$
L_{1}:(0,+\infty) \rightarrow \mathbb{R}, \quad L_{1}(\xi)=\xi-\frac{\delta_{u}}{\xi}, \quad L_{2}:(1,+\infty) \rightarrow \mathbb{R}, \quad L_{2}(\xi)=\xi-\frac{\delta_{u}}{\xi-\frac{1}{\xi}},
$$

we can express $y(1)=L_{2}^{-1} L_{1}\left(\lambda_{I}\right)$. As before, Lemma 3.2 applied to case $(b)$ completes the proof.

\section{$7 \quad$ Asymptotic behavior}

It is illustrative to compare the bounds obtained in the three above theorems for graphs with large index. Making the corresponding asymptotic developments, we have the following cases:

(a) Connection of an isolated vertex (to $g$ vertices):

$$
\lambda_{F} \leq H^{-1}\left(\lambda_{I}\right)=\lambda_{I}+\frac{g}{\lambda_{I}}+\mathrm{o}\left(\frac{1}{\lambda_{I}}\right)
$$

(b) Addition of an edge (between vertices of degrees $\delta_{u}, \delta_{v}$ ):

$$
\lambda_{F} \leq 1+K^{-1}\left(K\left(\lambda_{I}\right)-1\right)=\lambda_{I}+\frac{\delta_{u}+\delta_{v}}{\lambda_{I}^{2}}+\mathrm{o}\left(\frac{1}{\lambda_{I}^{2}}\right) .
$$

(c) Addition of a pendant edge (to a vertex of degree $\delta_{u}$ ):

$$
\lambda_{F} \leq L_{2}^{-1} L_{1}\left(\lambda_{I}\right)=\lambda_{I}+\frac{\delta_{u}}{\lambda_{I}^{3}}+\mathrm{o}\left(\frac{1}{\lambda_{I}^{3}}\right)
$$

Let us observe that the maximum possible variation in the spectral radius caused by the three perturbations considered are, for large $\lambda_{I}$, of different orders of magnitude.

Notice also that, by applying iteratively the above formulas, we can obtain asymptotic bounds for 'multiple perturbations'. For instance, if $G_{F}$ is obtained from $G_{I}$ by joining all the vertices $u_{1}, u_{2}, \ldots, u_{m}$ of a coclique, with respective degrees $\delta_{1}, \delta_{2}, \ldots, \delta_{m}$, we get, by applying the bound for the addition of an edge $\left(\begin{array}{c}m \\ 2\end{array}\right)$ times,

$$
\lambda_{F} \leq \lambda_{I}+\frac{(m-1) \sum_{i=1}^{m} \delta_{i}+(m-2)\left(\begin{array}{c}
m \\
2
\end{array}\right)}{\lambda_{I}^{2}}+\mathrm{o}\left(\frac{1}{\lambda_{I}^{2}}\right) .
$$


Acknowledgments. The authors are most grateful to Professor Peter Rowlinson and one of the referees for their useful comments and suggestions on the topic of this paper. Research supported by the Ministerio de Ciencia e Innovación, Spain, and the European Regional Development Fund under project MTM2008-06620-C03-01 and by the Catalan Research Council under project 2009SGR1387.

\section{References}

[1] F.K. Bell and P. Rowlinson, The change in index of a graph resulting from the attachment of a pendant edge, Proc. Roy. Soc. Edinburgh Sect. A 108 (1988), no. $1-2,67-74$.

[2] D. Cvetković, M. Doob, I. Gutman, and A. Torgašev, Recent Results in the Theory of Graph Spectra, Annals of Discrete Mathematics, North-Holland, Amsterdam, 1988.

[3] D. Cvetković, M. Doob, and H. Sachs, Spectra of Graphs. Theory and Application, Pure and Applied Mathematics 87, Academic Press, New York, 1979.

[4] C.D. Cvetković and P. Rowlinson, The largest eigenvalue of a graph: a survey, Linear and Multilinear Algebra 28 (1990), no. 1-2, 3-33.

[5] D. Cvetković and P. Rowlinson, Spectral graph theory, in: Topics in Algebraic Graph Theory (eds. L. W. Beineke and R. J. Wilson), Cambridge Univ. Press, Cambridge, 2004.

[6] C.D. Godsil, Algebraic Combinatorics, Chapman and Hall, New York, 1993.

[7] R.-C. Li, Matrix perturbation theory, in: Handbook of Linear Algebra, (eds. R. Brualdi, A. Greenbaum and R. Mathias), Chapman \& Hall/CRC, Boca Raton, FL, 2007.

[8] Ch. Maas, Perturbation results for the adjacency spectrum of a graph, Z. Angew. Math. Mech. 67 (1987), no. 5, T428-T430.

[9] P. Rowlinson, On angles and perturbations of graphs, Bull. London Math. Soc. 20 (1988), no. 3, 193-197.

[10] P. Rowlinson, Graph perturbations, in: Surveys in Combinatorics, 1991 (Guildford, 1991), 187-219, London Math. Soc. Lecture Note Ser. 166, Cambridge Univ. Press, Cambridge, 1991.

[11] P. Rowlinson, The spectrum of a graph modified by the addition of a vertex, Univ. Beograd. Publ. Elektrotehn. Fak. Ser. Mat. 3 (1992), 67-70.

[12] A.J. Schwenk and R.J. Wilson, On the eigenvalues of a graph, in: Selected Topics in Graph Theory, 307-336, (eds. L.W. Beineke and R.J. Wilson), Academic Press, London, 1978.

[13] G.W. Stewart and J. Sun, Matrix Perturbation Theory, Academic Press, Boston, MA, 1990.

[14] J. Szarski, Differential Inequalities, Monografie Matematyczne 43 Państwowe Wydawnictwo Naukowe, Warsaw, 1965. 